\title{
Kernel identities and vectorial regularisation
}

\author{
Christian Bargetz $^{1}$ (D) Norbert Ortner ${ }^{1}$
}

Received: 13 January 2017 / Accepted: 14 June 2017 / Published online: 5 July 2017

C The Author(s) 2017. This article is an open access publication

\begin{abstract}
We present the new method of "vectorial regularisation" to prove kernel identities. This method is applied to derive both known kernel identities, e.g. $\dot{\mathcal{B}}_{x y}=\dot{\mathcal{B}}_{x} \widehat{\otimes}_{\varepsilon} \dot{\mathcal{B}}_{y}, \mathcal{D}_{L^{1}, x y}^{\prime}=\mathcal{D}_{L^{1}, x}^{\prime} \widehat{\otimes}_{\pi} \mathcal{D}_{L^{1}, y}^{\prime}$, as well as new ones: $\dot{\mathcal{B}}_{x y}^{\prime}=\dot{\mathcal{B}}_{x}^{\prime} \widehat{\otimes}_{\varepsilon} \dot{\mathcal{B}}_{y}^{\prime}$ and $\mathcal{D}_{L^{1}, x y}=\mathcal{D}_{L^{1}, x} \widehat{\otimes}_{\pi} \mathcal{D}_{L^{1}, y}$.
\end{abstract}

Keywords Vector-valued distributions - Regularisation of distributions - Convolution of distributions $\cdot$ Completed topological tensor products

Mathematics Subject Classification 46F05 - 46F10

\section{Introduction}

Including the famous kernel theorems of L. Schwartz which can be rephrased as

$$
\mathcal{S}_{x y}^{\prime}=\mathcal{S}_{x}^{\prime} \widehat{\otimes} \mathcal{S}_{y}^{\prime} \text { and } \mathcal{D}_{x y}^{\prime}=\mathcal{D}_{x}^{\prime} \widehat{\otimes} \mathcal{D}_{y}^{\prime}
$$

in Proposition 28 in [18, p. 98] nine kernel identities are stated. In addition to these nine identities, Schwartz' $[18,19]$ treatise on vector-valued distributions contains the kernel identities

$$
\dot{\mathcal{B}}_{x y}=\dot{\mathcal{B}}_{x} \widehat{\otimes}_{\varepsilon} \dot{\mathcal{B}}_{y}, \quad \mathcal{D}_{L^{1}, x y}^{\prime}=\mathcal{D}_{L^{1}, x}^{\prime} \widehat{\otimes} \mathcal{D}_{L^{1}, y}^{\prime} \quad \text { and } \quad \mathcal{D}_{x y}=\mathcal{D}_{x} \widehat{\otimes}_{\iota} \mathcal{D}_{y}
$$

Communicated by A. Constantin.

Christian Bargetz

christian.bargetz@uibk.ac.at

1 Institut für Mathematik, Universität Innsbruck, Technikerstraße 13, 6020 Innsbruck, Austria 
The space $\mathcal{D}_{L^{1}}^{\prime}$ is the space of integrable distributions. The usefulness of the kernel identity for integrable distributions can be seen by considering the proofs for the equivalence of the following conditions for the convolvabilty of two distributions $S, T \in \mathcal{D}^{\prime}\left(\mathbb{R}^{n}\right)$. The convolvability condition

$$
\forall \varphi \in \mathcal{D}\left(\mathbb{R}^{n}\right): \varphi(x+y) S(x) T(y) \in \mathcal{D}_{L^{1}}^{\prime}\left(\mathbb{R}_{x y}^{2 n}\right)
$$

has been introduced by Schwartz [15, Exposé $\left.{ }^{\circ} 22\right]$ and has been rediscovered by Horváth [8]. The condition

$$
\forall \varphi \in \mathcal{D}\left(\mathbb{R}^{n}\right):(\varphi * \check{S}) T \in \mathcal{D}_{L^{1}}^{\prime}\left(\mathbb{R}^{n}\right)
$$

has been given by Schwartz [18, pp. 131-132]. Making use of the kernel identities above allows to show the equivalence of $(\mathrm{SH})$ and $(\mathrm{S})$ in a few lines-compare [14] to [21] or [12] to [13].

In addition, the convolvability condition

$$
\forall \varphi, \psi \in \mathcal{D}\left(\mathbb{R}^{n}\right):(\varphi * \check{S})(\psi * T) \in L^{1}\left(\mathbb{R}^{n}\right)
$$

or, equivalently,

$$
\forall \varphi, \psi \in \mathcal{D}\left(\mathbb{R}^{n}\right):(\varphi * \check{S})(\psi * T) \in \mathcal{D}_{L^{1}}\left(\mathbb{R}^{n}\right)
$$

was introduced by Chevalley [5]. It turns out that all these conditions are, in fact, equivalent. For a proof of the equivalence of $(\mathrm{S})$ and $(\mathrm{C})$ or $\left(\mathrm{C}^{\prime}\right)$ the kernel identities

$$
L_{x y}^{1}=L_{x}^{1} \widehat{\otimes}_{\pi} L_{y}^{1},
$$

which is due to Grothendieck, see [7, chap. I, pp. 61], or

$$
\mathcal{D}_{L^{1}, x y}=\mathcal{D}_{L^{1}, x} \widehat{\otimes}_{\pi} \mathcal{D}_{L^{1}, y}
$$

see Proposition 10, turn out to be a useful tool.

The spaces $\dot{\mathcal{B}}$ and $\dot{\mathcal{B}}^{\prime}$ appear naturally in the context of the question of whether derivatives can be swapped in a convolution product, i.e. the question of whether for distributions $S$ and $T$ on $\mathbb{R}^{n}$ the identity

$$
\left(\partial_{j} S\right) * T=S *\left(\partial_{j} T\right)
$$

holds provided that both convolution products exist. In [11] it is shown that this is true under the additional assumption that

$$
(\varphi * \check{S}) T \in \dot{\mathcal{B}}^{\prime}
$$

for all test functions $\varphi \in \mathcal{D}$. This condition generalises the classical result that (1) holds true for convolvable distributions. The kernel identity for distributions vanishing 
at infinity, see Proposition 4, can be used to give a short proof for the equivalence of the condition above with

$$
\varphi(x+y) S(x) T(y) \in \dot{\mathcal{B}}_{x y}^{\prime}
$$

for all test functions $\varphi \in \mathcal{D}$. Using the identity

$$
\dot{\mathcal{B}}_{x y}=\dot{\mathcal{B}}_{x} \widehat{\otimes}_{\varepsilon} \dot{\mathcal{B}}_{y}
$$

see Proposition 17 in [18], it can be shown that the above condition is equivalent to

$$
\forall \varphi, \psi \in \mathcal{D}:(\varphi * \check{S})(\psi * T) \in \dot{\mathcal{B}}
$$

which is an analogue of C. Chevalley's condition mentioned above.

Regularisation results play an important role in Schwartz' [20] classical monograph. The space $\mathcal{S}^{\prime}\left(\mathbb{R}^{n}\right)$ of temperate distributions is characterised by regularisation in Théorème VI, [20, p. 239], i.e., it is shown that a distribution $T \in \mathcal{D}^{\prime}\left(\mathbb{R}^{n}\right)$ is temperate if and only if $\varphi * T \in \mathcal{O}_{C}$ for all test functions $\varphi \in \mathcal{D}\left(\mathbb{R}^{n}\right)$. A similar characterisation of the space $\mathcal{O}_{C}^{\prime}\left(\mathbb{R}^{n}\right)$ is given in Théorème IX, [20, p. 244].

In the following all distribution spaces are defined on the whole of $\mathbb{R}^{n}$ and we suppress the domain in order to shorten the notation, i.e., we write $\mathcal{D}^{\prime}$ for $\mathcal{D}^{\prime}\left(\mathbb{R}^{n}\right)$, $\mathcal{D}_{L^{p}}^{\prime}$ for $\mathcal{D}_{L^{p}}^{\prime}\left(\mathbb{R}^{n}\right)$, etc.

Motivated by the characterisations by regularisation stated above, a regularisation property is a statement where the elements of a space of distributions are characterised by the behaviour of their regularisations. In other words, it is a statement of the following form: Let $1 \leq p \leq \infty$. For $T \in \mathcal{D}^{\prime}$ the assertions

1. $T \in \mathcal{D}_{L^{p}}^{\prime}$

2. $\forall \varphi \in \mathcal{D}: \varphi * T \in L^{p}$

are equivalent by Théorème XXV of [20, p. 201]. By means of the associated difference kernel

$$
T(x-y) \in \mathcal{D}_{x y}^{\prime}=\mathcal{D}^{\prime}\left(\mathbb{R}_{x}^{n} \times \mathbb{R}_{y}^{n}\right)
$$

the equivalence above can be translated into the equivalence

$$
T \in \mathcal{D}_{L^{p}}^{\prime} \Leftrightarrow T(x-y) \in \mathcal{D}_{y}^{\prime} \widehat{\otimes} L_{x}^{p} .
$$

The "associated difference kernel" $T(x-y)$ is defined in [18, pp. 103-104]. For a detailed discussion of this reformulation, we refer the reader to Proposition 3 of [12, p. 326]. An advantage of such a reformulation is that it no longer contains either test functions or quantifiers.

A vectorial regularisation property reads as: Let $E$ be a space of distributions and $K(x, z) \in \mathcal{D}_{x}^{\prime}\left(E_{z}\right)$. Then,

$$
K(x, z) \in \mathcal{D}_{L^{p}, x}^{\prime}\left(E_{z}\right) \Leftrightarrow K(x-y, z) \in\left(\mathcal{D}_{y}^{\prime} \widehat{\otimes} L_{x}^{p}\right)\left(E_{z}\right)
$$


The space $\mathcal{D}_{x}^{\prime}\left(E_{z}\right)$ of $E$-valued distributions is defined as the subspace $\mathcal{D}_{x}^{\prime} \varepsilon E_{z}$ of $\mathcal{D}_{x z}^{\prime}$ wherein the $\varepsilon$-product is defined in [18, p. 18]. Note that by Corollary 1 in [18, p. 47] $\mathcal{D}^{\prime} \varepsilon E=\mathcal{D}^{\prime} \widehat{\otimes}_{\varepsilon} E$ if $E$ is complete.

The symbol $\otimes$ without subscript is used if $\otimes_{\pi}=\otimes_{\varepsilon}$, e.g., if one of the spaces is nuclear.

If $\mathcal{D}_{L^{p}}^{\prime} \widehat{\otimes}_{\pi} E$ is used instead of $\mathcal{D}_{L^{p}}^{\prime} \widehat{\otimes}_{\varepsilon} E$ we speak of vectorial regularisation with the completed projective tensor product.

By kernel identities, we understand statements as e.g. L. Schwartz's classical kernel theorem, i.e., $\mathcal{D}_{x y}^{\prime}=\mathcal{D}_{x}^{\prime} \widehat{\otimes} \mathcal{D}_{y}^{\prime}$. Two fundamental examples of kernel identities are given in $[7$, chap. I, pp. 61, 90]:

$$
L^{1}(X) \widehat{\otimes}_{\pi} L^{1}(Y)=L^{1}(X \times Y) \text { and } \mathcal{C}_{0}(X) \widehat{\otimes}_{\varepsilon} \mathcal{C}_{0}(Y)=\mathcal{C}_{0}(X \times Y),
$$

where $X$ and $Y$ are locally compact spaces. In order to abbreviate the notation, we will write these identities for $X=\mathbb{R}^{n}$ and $Y=\mathbb{R}^{m}$ as

$$
L_{x}^{1} \widehat{\otimes}_{\pi} L_{y}^{1}=L_{x y}^{1} \quad \text { and } \quad \mathcal{C}_{0, x} \widehat{\otimes}_{\varepsilon} \mathcal{C}_{0, y}=\mathcal{C}_{0, x y}
$$

Schwartz [18] found the algebraic and topological kernel identities

$$
\mathcal{D}_{L^{1}, x}^{\prime} \widehat{\otimes}_{\pi} \mathcal{D}_{L^{1}, y}^{\prime}=\mathcal{D}_{L^{1}, x y}^{\prime}
$$

for integrable distributions (Proposition 38 in [18, p. 135]) and

$$
\dot{\mathcal{B}}_{x} \widehat{\otimes}_{\varepsilon} \dot{\mathcal{B}}_{y}=\dot{\mathcal{B}}_{x y}
$$

for smooth functions with derivatives vanishing at infinity (Proposition 17 in [18, p. 59]). As mentioned above, further kernel identities are given in Proposition 28 in [18, p. 98],

$$
\begin{array}{ll}
\mathcal{E}_{x y}=\mathcal{E}_{x} \widehat{\otimes} \mathcal{E}_{y}, & \mathcal{S}_{x y}=\mathcal{S}_{x} \widehat{\otimes} \mathcal{S}_{y}, \\
\mathcal{E}_{x y}^{\prime}=\mathcal{E}_{x}^{\prime} \widehat{\otimes} \mathcal{E}_{y}^{\prime}, & \mathcal{S}_{x y}^{\prime}=\mathcal{S}_{x}^{\prime} \widehat{\otimes} \mathcal{S}_{y}^{\prime}
\end{array}
$$

Note that however,

$$
\mathcal{D}_{x y}=\mathcal{D}_{x} \widehat{\otimes}_{\iota} \mathcal{D}_{y} \quad \text { and } \quad \mathcal{O}_{C, x y}=\mathcal{O}_{C, x} \widehat{\otimes}_{\iota} \mathcal{O}_{C, y}
$$

by Proposition 1 bis. in [19, p. 17] and [4, p. 34], respectively. Here $\otimes_{\iota}$ denotes the inductive tensor product defined in Definition 3 in [7, Chap. I, p. 74] and $\widehat{\otimes}_{\iota}$ its completion.

Concerning the applicability of such identities let us mention that, e.g., (3) is used to prove the equivalence of the two different convolvability conditions for distributions $S, T \in \mathcal{D}^{\prime}:$

$$
S(x-y) T(y) \in \mathcal{D}_{x}^{\prime} \widehat{\otimes} \mathcal{D}_{L^{1}, y}^{\prime}
$$


and

$$
\delta(z-x-y) S(x) T(y) \in \mathcal{D}_{z}^{\prime} \widehat{\otimes} \mathcal{D}_{L^{1}, x y}^{\prime}
$$

in [12, p. 324], [14, p. 195] and [21, p. 27].

The aim of this article is to prove, in a uniform manner, known and new kernel identities by vectorial regularisation properties. In Proposition 9 we give a new proof of the kernel identity (3) and in Proposition 3 an new proof of (4). The new identity

$$
\mathcal{D}_{L^{1}, x y}=\mathcal{D}_{L^{1}, x} \widehat{\otimes}_{\pi} \mathcal{D}_{L^{1}, y}
$$

is shown in Proposition 10 and the identity

$$
\dot{\mathcal{B}}_{x y}^{\prime}=\dot{\mathcal{B}}^{\prime} \widehat{\otimes}_{\varepsilon} \dot{\mathcal{B}}_{y}^{\prime}
$$

in Proposition 4. Our proofs of the identities $(3,4,6,7)$ show that they are all consequences of Grothendieck's fundamental examples (2).

Also it turns out that in some cases the topological part of the kernel identities follows from the algebraic identity and abstract structural results, e.g. for complete spaces of distributions $\mathcal{H}$ the continuous embeddings

$$
\mathcal{H}_{x} \widehat{\otimes}_{\varepsilon} \mathcal{H}_{y} \hookrightarrow \mathcal{D}_{x}^{\prime} \widehat{\otimes}_{\varepsilon} \mathcal{D}_{y}^{\prime}=\mathcal{D}_{x y}^{\prime} \hookleftarrow \mathcal{H}_{x y}
$$

imply that the identity mapping $\mathcal{H}_{x y} \rightarrow \mathcal{H}_{x} \widehat{\otimes}_{\varepsilon} \mathcal{H}_{y}$ has a closed graph. In concrete cases, sequence-space representations can be used to check whether these spaces satisfy the assumptions of a suitable closed graph theorem.

We use the notations of Schwartz [20], e.g. the space of distributions $\mathcal{E}, \mathcal{E}^{\prime}, \mathcal{D}, \mathcal{D}^{\prime}$, $\mathcal{D}_{L^{p}}, \mathcal{D}_{L^{p}}^{\prime}$ for $1 \leq p \leq \infty, \dot{\mathcal{B}}$ and $\dot{\mathcal{B}}^{\prime}$ (which is not the dual of $\dot{\mathcal{B}}$ but the closure of $\mathcal{E}^{\prime}$ in $\left.\mathcal{D}_{L^{\infty}}^{\prime}\right)$. For vector-valued distributions, constant use is made of Schwartz' $[18,19]$ treatise. Instead of $K(\hat{x}, \hat{y})$ we simply write $K(x, y)$ for kernels $K(x, y) \in \mathcal{D}_{x y}^{\prime}$.

Proposition 4 was presented in a talk given by the second author in Vienna, June, 2015.

\section{Regularisation and the injective tensor product}

Recall that by remarque $3^{\circ}$ in [20, p. 202] distributions vanishing at infinity can be characterised by regularisation properties, i.e. a distribution $S \in \mathcal{D}^{\prime}$ is an element of $\dot{\mathcal{B}}^{\prime}$ if and only if one of the following equivalent conditions is satisfied:

1. $\forall \varphi \in \mathcal{D}: \varphi * S \in \dot{\mathcal{B}}$

2. $\forall \varphi \in \mathcal{D}: \varphi * S \in \mathcal{C}_{0}$

3. $S(x-y) \in \mathcal{D}_{y}^{\prime} \widehat{\otimes} \dot{\mathcal{B}}_{x}$

4. $S(x-y) \in \mathcal{D}_{y}^{\prime} \widehat{\otimes} \mathcal{C}_{0, x}$.

The following proposition can be understood as a generalisation of these regularisation properties to kernels. 
Proposition 1 Let $E$ be a complete space of distributions. For a kernel $K(x, z) \in \mathcal{D}_{x z}^{\prime}$ the following characterizations hold:

1. $K(x, z) \in \dot{\mathcal{B}}_{x}^{\prime} \widehat{\otimes}_{\varepsilon} E_{z} \Leftrightarrow K(x-y, z) \in\left(\mathcal{D}_{y}^{\prime} \widehat{\otimes} \mathcal{C}_{0, x}\right) \widehat{\otimes}_{\varepsilon} E_{z}$.

2. $K(x, z) \in \dot{\mathcal{B}}_{x} \widehat{\otimes}_{\varepsilon} E_{z} \Leftrightarrow K(x-y, z) \in\left(\mathcal{E}_{y} \widehat{\otimes} \mathcal{C}_{0, x}\right) \widehat{\otimes}_{\varepsilon} E_{z}$.

Although for a normal space of distributions $E$ the characterization 1 of this Proposition is a special case of Proposition 15 in [3], we include it nevertheless to keep the article self-contained.

Proof 1. We first show the case of distributions.

$\Rightarrow$ : The mapping

$$
\tau: \dot{\mathcal{B}}^{\prime} \rightarrow \mathcal{D}_{y}^{\prime} \widehat{\otimes} \mathcal{C}_{0, x}, S \mapsto S(x-y)
$$

is well-defined, linear and continuous according to Remarque 3 in [20, p. 202]. Hence also the mapping

$$
\tau \varepsilon \operatorname{id}_{E}: \dot{\mathcal{B}}_{x}^{\prime} \widehat{\otimes}_{\varepsilon} E_{z} \rightarrow\left(\mathcal{D}_{y}^{\prime} \widehat{\otimes} \mathcal{C}_{0, x}\right) \widehat{\otimes}_{\varepsilon} E_{z}, K(x, z) \mapsto K(x-y, z)
$$

as by Proposition 1 in [18, p. 20] the $\varepsilon$-product of continuous linear mappings is again continuous.

$\Leftarrow$ : Multiplication of $K(x-y, z) \in \mathcal{D}_{y}^{\prime}\left(\mathcal{C}_{0, x} \widehat{\otimes}_{\varepsilon} E_{z}\right)$ by $\delta(w-y) \in \mathcal{D}_{y} \widehat{\otimes} \mathcal{D}_{w}^{\prime}$ using Proposition 25 in [19, p. 120] leads to

$\delta(w-y) K(x-y, z) \in \mathcal{E}_{y}^{\prime}\left(\mathcal{D}_{w}^{\prime} \widehat{\otimes}\left(\mathcal{C}_{0, x} \widehat{\otimes}_{\varepsilon} E_{z}\right)\right)=\left(\mathcal{E}_{y}^{\prime} \widehat{\otimes}_{\varepsilon} \mathcal{C}_{0, x}\right) \widehat{\otimes}_{\varepsilon}\left(\mathcal{D}_{w}^{\prime} \widehat{\otimes}_{\varepsilon} E_{z}\right)$

From $\mathcal{C}_{0, x} \widehat{\otimes}_{\varepsilon} \mathcal{E}_{y}^{\prime} \hookrightarrow \dot{\mathcal{B}}_{x y}^{\prime}$ and the invariance of $\dot{\mathcal{B}}_{x, y}^{\prime}$ under the coordinate transform

$$
\begin{array}{rlrl}
x-y & =u & x & =u \\
y & =v & y & =v
\end{array}
$$

we deduce

$$
\delta(v-w) K(u, z) \in \dot{\mathcal{B}}_{u v}^{\prime} \widehat{\otimes}_{\varepsilon} \mathcal{D}_{w}^{\prime} \widehat{\otimes}_{\varepsilon} E_{z} \subset\left(\mathcal{S}_{v}^{\prime} \widehat{\otimes}_{\mathcal{B}_{u}^{\prime}}^{\prime}\right)\left(\mathcal{D}_{w}^{\prime} \widehat{\otimes}_{\varepsilon} E_{z}\right)
$$

Evaluation with $\mathrm{e}^{-|v|^{2}} \in \mathcal{S}_{v}$ yields

$$
\mathrm{e}^{-|w|^{2}} K(u, z) \in \dot{\mathcal{B}}_{u}^{\prime} \widehat{\otimes}_{\varepsilon} \mathcal{D}_{w}^{\prime} \widehat{\otimes}_{\varepsilon} E_{z}=\mathcal{D}_{w}^{\prime}\left(\dot{\mathcal{B}}_{u}^{\prime} \widehat{\otimes}_{\varepsilon} E_{z}\right)
$$

Multiplication by $\mathrm{e}^{|w|^{2}} \in \mathcal{E}_{w}$, which is possible by Theorem 7.1 in [17], leads to

$$
K(u, z) \in \mathcal{D}_{w}^{\prime}\left(\dot{\mathcal{B}}_{u}^{\prime} \widehat{\otimes}_{\varepsilon} E_{z}\right)
$$


and hence

$$
K(u, z) \in \dot{\mathcal{B}}_{u}^{\prime} \widehat{\otimes}_{\varepsilon} E_{z}
$$

2. The implication " $\Rightarrow$ " is completely analogous to the case of distributions if we use that the convolution mapping $*: \mathcal{E}^{\prime} \times \dot{\mathcal{B}} \rightarrow \mathcal{C}_{0}$ is well defined and hypocontinuous since $\mathcal{E}^{\prime} \hookrightarrow \mathcal{D}_{L^{1}}^{\prime}$ and $\dot{\mathcal{B}} \hookrightarrow \mathcal{C}_{0}$ (see e.g. [10]).

Let us show the implication " $\Leftarrow$ ". The vectorial scalar product of

$$
K(x-y, z) \in \mathcal{E}_{y}\left(\mathcal{C}_{0, x} \widehat{\otimes}_{\varepsilon} E_{z}\right)
$$

with $\partial^{\alpha} \delta(y) \in \mathcal{E}_{y}^{\prime}$ yields $\partial_{x}^{\alpha} K(x, z) \in \mathcal{C}_{0, x} \widehat{\otimes}_{\varepsilon} E_{z}$ for all $\alpha \in \mathbb{N}_{0}^{n}$. From this we deduce $K(x, z) \in \dot{\mathcal{B}}_{x} \widehat{\otimes}_{\varepsilon} E_{z}$ using the compatibility of the vector-valued scalar product with continuous linear mappings by [19, p. 18].

Remark 2 Note that it is possible to generalize this result to non-complete spaces of distributions but in this case the completed $\varepsilon$-tensor product has to be replaced by the $\varepsilon$-product.

Proposition 3 (see Proposition 17 in [18]) The space of smooth functions vanishing at infinity satisfies the kernel identity

$$
\dot{\mathcal{B}}_{x y}=\dot{\mathcal{B}}_{x} \widehat{\otimes}_{\varepsilon} \dot{\mathcal{B}}_{y}
$$

algebraically and topologically.

Proof In order to show the algebraic part, observe that for $K(x, y) \in \mathcal{D}_{x y}^{\prime}$ we get

$$
\begin{aligned}
K(x, y) \in \dot{\mathcal{B}}_{x y} & \Leftrightarrow K(x-z, y-w) \in \mathcal{E}_{z w} \widehat{\otimes}_{\varepsilon} \mathcal{C}_{0, x y}=\left(\mathcal{E}_{z} \widehat{\otimes} \mathcal{C}_{0, x}\right) \widehat{\otimes}_{\varepsilon}\left(\mathcal{E}_{w} \widehat{\otimes} \mathcal{C}_{0, y}\right) \\
& \Leftrightarrow K(x, y-w) \in \dot{\mathcal{B}}_{x}\left(\mathcal{E}_{w} \widehat{\otimes} \mathcal{C}_{0, y}\right) \\
& \Leftrightarrow K(x, y) \in \dot{\mathcal{B}}_{x} \widehat{\otimes}_{\varepsilon} \dot{\mathcal{B}}_{y} .
\end{aligned}
$$

Note that the first two equivalences follow from the kernel identities for $\mathcal{E}$ and $\mathcal{C}_{0}$ since the convolution

$$
\dot{\mathcal{B}} \times \mathcal{E}^{\prime} \rightarrow \dot{\mathcal{B}} \subset \mathcal{C}_{0}
$$

is well-defined. The last equivalence is a consequence of Proposition 1.

We are therefore left to show the topological identity. As the $\varepsilon$-product of two continuous linear mappings is again continuous, we see that the mapping

$$
\dot{\mathcal{B}}_{x} \widehat{\otimes}_{\varepsilon} \dot{\mathcal{B}}_{y} \rightarrow \mathcal{C}_{0, x} \widehat{\otimes}_{\varepsilon} \mathcal{C}_{0, y}=\mathcal{C}_{0, x y}, f \mapsto \partial_{x}^{\alpha} \partial_{y}^{\beta} f
$$

is continuous for all multi-indices $\alpha$ and $\beta$. Therefore the topology of $\dot{\mathcal{B}}_{x} \widehat{\otimes}_{\varepsilon} \dot{\mathcal{B}}_{y}$ is finer than the one of $\dot{\mathcal{B}}_{x y}$. Hence, being comparable Fréchet space topologies on the same vector space, these topologies coincide by the closed graph theorem. 
Proposition 4 The space of distributions vanishing at infinity satisfies the kernel identity

$$
\dot{\mathcal{B}}_{x y}^{\prime}=\dot{\mathcal{B}}_{x}^{\prime} \widehat{\otimes}_{\varepsilon} \dot{\mathcal{B}}_{y}^{\prime}
$$

algebraically and topologically.

Proof For $K(x, y) \in \mathcal{D}_{x y}^{\prime}$ we start with the characterization

$$
K(x, y) \in \dot{\mathcal{B}}_{x y}^{\prime} \Leftrightarrow K(x-z, y-w) \in \mathcal{D}_{z w}^{\prime} \widehat{\otimes} \dot{\mathcal{B}}_{x y}
$$

of $\dot{\mathcal{B}}^{\prime}$ by regularisation. From this we deduce

$$
\begin{aligned}
K(x, y) \in \dot{\mathcal{B}}_{x y}^{\prime} & \Leftrightarrow K(x-z, y-w) \in\left(\mathcal{D}_{z}^{\prime} \widehat{\otimes} \mathcal{D}_{w}^{\prime}\right) \widehat{\otimes}\left(\dot{\mathcal{B}}_{x} \widehat{\otimes}_{\varepsilon} \dot{\mathcal{B}}_{y}\right) \\
& \Leftrightarrow K(x-z, y-w) \in\left(\mathcal{D}_{z}^{\prime} \widehat{\otimes} \dot{\mathcal{B}}_{x}\right) \widehat{\otimes}_{\varepsilon}\left(\mathcal{D}_{w}^{\prime} \widehat{\otimes}_{\mathcal{B}_{y}}\right)
\end{aligned}
$$

using the kernel theorem for $\dot{\mathcal{B}}$ and $\mathcal{D}^{\prime}$ as well as the commutativity of the $\varepsilon$-tensor product. From this we obtain by Proposition 1,

$$
\begin{aligned}
K(x, y) \in \dot{\mathcal{B}}_{x y}^{\prime} & \Leftrightarrow K(x, y-w) \in \dot{\mathcal{B}}_{x}^{\prime} \widehat{\otimes}_{\varepsilon}\left(\mathcal{D}_{w}^{\prime} \widehat{\otimes}_{\dot{\mathcal{B}}}\right)=\left(\mathcal{D}_{w}^{\prime} \widehat{\otimes}_{\dot{\mathcal{B}}}\right) \widehat{\otimes}_{\varepsilon} \dot{\mathcal{B}}_{x}^{\prime} \\
& \Leftrightarrow K(x, y) \in \dot{\mathcal{B}}_{y}^{\prime} \widehat{\otimes}_{\varepsilon} \dot{\mathcal{B}}_{x}^{\prime}
\end{aligned}
$$

which proves the algebraic part of the kernel identity. Using the sequence space representation $\dot{\mathcal{B}}^{\prime}=s^{\prime} \widehat{\otimes} c_{0}$ given in Theorem 3 in [2, p. 13] and

$$
\left(s^{\prime} \widehat{\otimes}_{\varepsilon} c_{0}\right) \widehat{\otimes}_{\varepsilon}\left(s^{\prime} \widehat{\otimes}_{\varepsilon} c_{0}\right) \cong\left(s^{\prime} \widehat{\otimes} s^{\prime}\right) \widehat{\otimes}\left(c_{0} \widehat{\otimes}_{\varepsilon} c_{0}\right) \cong s^{\prime} \widehat{\otimes} c_{0}
$$

we see by Proposition 7 in [2, p. 13] that both $\dot{\mathcal{B}}_{x y}^{\prime}$ and $\dot{\mathcal{B}}_{x}^{\prime} \widehat{\otimes} \dot{\mathcal{B}}_{y}^{\prime}$ are complete ultrabornological (DF)-spaces. From the continuity of the embeddings

$$
\dot{\mathcal{B}}_{x}^{\prime} \widehat{\otimes}_{\varepsilon} \dot{\mathcal{B}}_{y}^{\prime} \hookrightarrow \mathcal{D}_{x}^{\prime} \widehat{\otimes} \mathcal{D}_{y}^{\prime}=\mathcal{D}_{x y}^{\prime} \hookleftarrow \dot{\mathcal{B}}_{x y}^{\prime},
$$

we deduce that the identity mapping $\dot{\mathcal{B}}_{x}^{\prime} \widehat{\otimes}_{\varepsilon} \dot{\mathcal{B}}_{y}^{\prime} \rightarrow \dot{\mathcal{B}}_{x, y}^{\prime}$ has a closed graph. Therefore the topological identity follows by de Wilde's closed graph theorem (Theorem 5.4.1 in [9, p. 92]) since complete (DF)-spaces have a completing web by Proposition 12.4.6 in $[9$, p. 260].

\section{Regularisation and the projective tensor product}

In order to prove a version of Proposition 1 for the projective tensor product, we need the following lemma.

Lemma 5 For $1<q<\infty$ the following continuous embeddings hold:

$$
\mathcal{S}_{x} \widehat{\otimes} \mathcal{D}_{L^{q}, y} \hookrightarrow \mathcal{D}_{L^{q}, x y} \hookrightarrow \mathcal{E}_{x} \widehat{\otimes} \mathcal{D}_{L^{q}, y},
$$


i.e., theses spaces are contained with a finer topology. Moreover these spaces are contained as dense subspaces.

Proof From $\mathcal{E}_{x y}=\mathcal{E}_{x} \widehat{\otimes} \mathcal{E}_{y}$, we deduce that $\mathcal{D}_{L^{q}, x} \widehat{\otimes} \mathcal{E}_{y}$ is a space of smooth functions. Using Lebesgue's theorem on dominated convergence we conclude that for $f \in \mathcal{D}_{L^{q}, x y}$ the function $\mathbb{R}_{x}^{d} \rightarrow \mathcal{D}_{L^{q}, y}, x \mapsto f(x, \cdot)$ has continuous derivatives of all order. Continuity of the embedding $\mathcal{D}_{L^{q}, x y} \hookrightarrow \mathcal{E}_{x} \widehat{\otimes} \mathcal{D}_{L^{q}, y}$ follows inductively from the Sobolev trace theorem, see, e.g., Theorem 5.36 in [1].

Given $f \in \mathcal{S}_{x} \widehat{\otimes} \mathcal{D}_{L^{q}, y}$, the inequality

$$
\begin{aligned}
& \int_{\mathbb{R}^{d_{1}+d_{2}}}|f(x, y)|^{q} \mathrm{~d} x \mathrm{~d} y \\
& =\int_{\mathbb{R}^{d_{1}+d_{2}}}\left(1+|x|^{2}\right)^{-d_{1}-1}\left(1+|x|^{2}\right)^{d_{1}+1}|f(x, y)|^{q} \mathrm{~d} x \mathrm{~d} y \\
& \leq \int_{\mathbb{R}^{d_{1}}}\left(1+|x|^{2}\right)^{-d_{1}-1} \mathrm{~d} x \sup _{x \in \mathbb{R}^{d_{1}}} \int_{\mathbb{R}^{d_{2}}}\left(1+|x|^{2}\right)^{d_{1}+1}|f(x, y)|^{q} \mathrm{~d} y \\
& \leq C \sup _{x \in \mathbb{R}^{d_{2}}}\left(1+|x|^{2}\right)^{d_{1}+1} \int_{\mathbb{R}^{d_{2}}}|f(x, y)|^{q} \mathrm{~d} y
\end{aligned}
$$

proves $\mathcal{S}_{x} \widehat{\otimes} \mathcal{D}_{L^{q}, y} \hookrightarrow \mathcal{D}_{L^{q}, x y}$. The spaces are contained as dense subspaces since

$$
\mathcal{D}_{x y} \hookrightarrow \mathcal{D}_{x} \widehat{\otimes} \mathcal{D}_{y} \subset \mathcal{S}_{x} \widehat{\otimes} \mathcal{D}_{L^{q}, y}
$$

and the injective tensor product preserves dense subspaces by Proposition 16.2.5 in [9, p. 349].

Remark 6 A different proof of Lemma 5 can be given by using the representation of elements of completed $\pi$-tensor products of Fréchet spaces and the closed graph theorem.

Proposition 7 Let $E$ be a space of distributions and $1 \leq p<\infty$. For $K(x, z) \in \mathcal{D}_{x z}^{\prime}$ the following characterizations hold:

1. $K(x, z) \in \mathcal{D}_{L^{p}, x}^{\prime} \widehat{\otimes}_{\pi} E_{z} \Leftrightarrow K(x-y, z) \in\left(\mathcal{D}_{y}^{\prime} \widehat{\otimes} L_{x}^{p}\right) \widehat{\otimes}_{\pi} E_{z}$.

2. $K(x, z) \in \mathcal{D}_{L^{p}, x} \widehat{\otimes}_{\pi} E_{z} \Leftrightarrow K(x-y, z) \in\left(\mathcal{E}_{y} \widehat{\otimes} L_{x}^{p}\right) \widehat{\otimes}_{\pi} E_{z}$.

Proof 1. $\quad \Rightarrow$ : The mapping $\tau: \mathcal{D}_{L^{p}}^{\prime} \rightarrow \mathcal{D}_{y}^{\prime} \widehat{\otimes} L_{x}^{p}, S \mapsto S(x-y)$ is well-defined, linear and continuous according to [20, p. 204]. Hence also

$$
\tau \otimes \operatorname{id}_{E}: \mathcal{D}_{L^{p}, x}^{\prime} \widehat{\otimes}_{\pi} E_{z} \rightarrow\left(\mathcal{D}_{y}^{\prime} \widehat{\otimes} L_{x}^{p}\right) \widehat{\otimes}_{\pi} E_{z}, K(x, z) \mapsto K(x-y, z)
$$

as the $\pi$-tensor product of continuous linear mappings is again a continuous and linear mapping. 
$\Leftarrow$ : Multiplication of $K(x-y, z) \in \mathcal{D}_{y}^{\prime}\left(L_{x}^{p} \widehat{\otimes}_{\pi} E_{z}\right)$ with $\delta(w-y) \in \mathcal{D}_{y} \widehat{\otimes} \mathcal{D}_{w}^{\prime}$ according to Proposition 25 in [19, p. 120] yields

$$
\begin{aligned}
\delta(w-y) K(x-y, z) \in \mathcal{E}_{y}^{\prime}\left(L_{x}^{p} \widehat{\otimes}_{\pi}\left(\mathcal{D}_{w}^{\prime} \widehat{\otimes}_{\pi} E_{z}\right)\right) & =\mathcal{E}_{y}^{\prime} \widehat{\otimes}_{\pi} L_{x}^{p} \widehat{\otimes}_{\pi} \mathcal{D}_{w}^{\prime} \widehat{\otimes}_{\pi} E_{z} \\
& =\mathcal{D}_{w}^{\prime}\left(\mathcal{E}_{y}^{\prime}\left(L_{x}^{p}\right)\right) \widehat{\otimes}_{\pi} E_{z} \\
& \subset\left(\mathcal{D}_{w}^{\prime} \widehat{\otimes}_{L^{p}, x y}^{\prime}\right) \widehat{\otimes}_{\pi} E_{z} .
\end{aligned}
$$

The proof of the inclusion $L^{p} \widehat{\otimes} \mathcal{E}^{\prime} \subset \mathcal{D}_{L^{p}}^{\prime}$ uses the following argument: from $L^{p} \subset \mathcal{D}_{L^{p}}^{\prime}$, we obtain $L^{p} \widehat{\otimes} \mathcal{E}^{\prime} \subset \mathcal{D}_{L^{p}}^{\prime} \widehat{\otimes} \mathcal{E}^{\prime}$ since the injective tensor product preserves continuous embeddings. In order to see that the latter space is contained in $\mathcal{D}_{L^{p}}^{\prime}$, we distinguish two cases. For $p=1$ we observe that $\dot{\mathcal{B}}_{x y}=\dot{\mathcal{B}}_{x} \widehat{\otimes}_{\varepsilon} \dot{\mathcal{B}}_{y} \hookrightarrow \dot{\mathcal{B}}_{x} \widehat{\otimes} \mathcal{E}_{y}$ and for $p>1$ we use Lemma 5 to obtain $\mathcal{D}_{L^{q}} \subset \mathcal{E}_{x} \widehat{\otimes} \mathcal{D}_{L^{q}}$. Using these observations we arrive at the above inclusion by Théorème 12 in [7, chap. II, p. 76] which implies $(\dot{\mathcal{B}} \widehat{\otimes} \mathcal{E})^{\prime}=\mathcal{D}_{L^{1}, x}^{\prime} \widehat{\otimes}_{\mathcal{E}_{y}^{\prime}}^{\prime}$ and $\left(\mathcal{D}_{L^{q}} \widehat{\otimes} \mathcal{E}\right)^{\prime}=\mathcal{D}_{L^{p}}^{\prime} \widehat{\otimes} \mathcal{E}^{\prime}$ for $1<p<\infty$ since $\mathcal{D}_{L^{p}}, \dot{\mathcal{B}}$ and $\mathcal{E}$ are Fréchet spaces. The inclusion $\mathcal{E}_{y}^{\prime}\left(L_{x}^{p}\right) \subset \mathcal{D}_{L^{p}, x y}^{\prime}$ above follows by density and duality since

$$
\mathcal{D}_{L^{q}, x y} \subset \mathcal{D}_{L^{q}, x} \widehat{\otimes}_{\varepsilon} \mathcal{D}_{L^{q}, y} \subset \mathcal{D}_{L^{q}, x} \widehat{\otimes} \mathcal{E}_{y},
$$

where the first inclusion is a consequence of [6, p. 98], for $q$ given by $\frac{1}{p}+\frac{1}{q}=1$. Using the coordinate transform

$$
\begin{array}{rlrl}
x-y & =u & x & =u+v \\
y & =v & y & =v
\end{array}
$$

we obtain

$$
\delta(w-v) K(u, z) \in \mathcal{D}_{L^{p}, u, v}^{\prime} \widehat{\otimes}_{\pi}\left(\mathcal{D}_{w}^{\prime} \widehat{\otimes}_{\pi} E_{z}\right)
$$

from the invariance of $\mathcal{D}_{L^{p}, x, y}^{\prime}$ under coordinate transforms. From $\mathcal{D}_{L^{p}, x, y}^{\prime} \subset \mathcal{D}_{L^{p}, x}^{\prime} \widehat{\otimes} \mathcal{S}_{y}^{\prime}$ we deduce that the application of

$$
\delta(w-v) K(u, z) \in \mathcal{S}_{v}^{\prime}\left(\mathcal{D}_{w}^{\prime} \widehat{\otimes}\left(\mathcal{D}_{L^{p}, u}^{\prime} \widehat{\otimes}_{\pi} E_{z}\right)\right)
$$

to $\mathrm{e}^{-|v|^{2}} \in \mathcal{S}_{v}$ is

$$
\mathrm{e}^{-|w|^{2}} K(u, z) \in \mathcal{D}_{w}^{\prime} \widehat{\otimes}\left(\mathcal{D}_{L^{p}, u}^{\prime} \widehat{\otimes}_{\pi} E_{z}\right)
$$

Multiplication by $\mathrm{e}^{|w|^{2}} \in \mathcal{E}_{w}$ according to Theorem 7.1 in [17, p. 31] yields $K(u, z) \in \mathcal{D}_{w}^{\prime} \widehat{\otimes}\left(\mathcal{D}_{L^{p}, u}^{\prime} \widehat{\otimes}_{\pi} E_{z}\right)$ and hence $K(u, z) \in \mathcal{D}_{L^{p}, u}^{\prime} \widehat{\otimes}_{\pi} E_{z}$. 
2. The implication " $\Rightarrow$ " is completely analogous to the case of distributions if we use that the convolution mapping $*: \mathcal{E}^{\prime} \times \mathcal{D}_{L^{p}} \rightarrow L^{p}$ is well defined and hypocontinuous since $\mathcal{E}^{\prime} \hookrightarrow \mathcal{D}_{L^{1}}^{\prime}$ and $\mathcal{D}_{L^{p}} \hookrightarrow L^{p}$ (see e.g. [10]).

Let us show the implication " $\Leftarrow$ ". The vectorial scalar product of

$$
K(x-y, z) \in \mathcal{E}_{y} \widehat{\otimes}\left(L_{x}^{p} \widehat{\otimes}_{\pi} E_{z}\right)
$$

with $\partial^{\alpha} \delta(y) \in \mathcal{E}_{y}^{\prime}$ yields $\partial_{x}^{\alpha} K(x, z) \in L_{x}^{p} \widehat{\otimes}_{\pi} E_{z}$ for all $\alpha \in \mathbb{N}_{0}^{n}$. From this we deduce $K(x, z) \in \mathcal{D}_{L^{p}, x} \widehat{\otimes}_{\pi} E_{z}$ using the compatibility of the vector-valued scalar product with continuous linear mappings by [19, p. 18].

Remark 8 More general, the proof of equivalence 1 in Proposition 7 also works in the following situation. Let $\mathcal{H}^{\prime}$ be a space of distributions and $\mathcal{K}$ a space of functions such that the convolution mapping $\mathcal{H}^{\prime} \times \mathcal{D} \rightarrow \mathcal{K}$ is hypocontinuous. If additionally the embeddings

$$
\mathcal{K}_{x} \widehat{\otimes} \mathcal{E}_{y}^{\prime} \hookrightarrow \mathcal{H}_{x, y}^{\prime} \hookrightarrow \mathcal{H}_{x}^{\prime} \widehat{\otimes} \mathcal{S}_{y}^{\prime}
$$

are well-defined and continuous, for kernels $K(x, y) \in \mathcal{D}_{x, y}^{\prime}$ we get the following equivalence

$$
K(x, z) \in \mathcal{H}_{x}^{\prime} \widehat{\otimes}_{\pi} E_{z} \Leftrightarrow K(x-y, z) \in\left(\mathcal{D}_{y}^{\prime} \widehat{\otimes}_{\mathcal{K}}\right) \widehat{\otimes}_{\pi} E_{z} .
$$

Examples of spaces $\mathcal{H}^{\prime}$ satisfying condition (8) are duals of normal spaces of distributions $\mathcal{H}$ where the embeddings

$$
\mathcal{S}_{x} \widehat{\otimes} \mathcal{H}_{y} \hookrightarrow \mathcal{H}_{x, y} \hookrightarrow \mathcal{E}_{x} \widehat{\otimes} \mathcal{H}_{y}
$$

are well-defined and continuous. Note that the spaces $\mathcal{S} \widehat{\otimes} \mathcal{H}$ and $\mathcal{E} \widehat{\otimes} \mathcal{H}$ are spaces of $\mathcal{H}$-valued smooth functions. We refer to [16] for a detailed treatment of these spaces.

In the following we will discuss two kernel-identities as applications of Proposition 7.

Proposition 9 (see Proposition 38 in [18, p. 135]) The space of integrable distributions satisfies the kernel identity

$$
\mathcal{D}_{L^{1}, x, y}^{\prime}=\mathcal{D}_{L^{1}, x}^{\prime} \widehat{\otimes}_{\pi} \mathcal{D}_{L^{1}, y}^{\prime}
$$

algebraically and topologically.

Proof For $K(x, y) \in \mathcal{D}_{x, y}^{\prime}$ we have the equivalence

$$
K(x, y) \in \mathcal{D}_{L^{1}, x, y}^{\prime} \Leftrightarrow K(x-z, y-w) \in \mathcal{D}_{z, w}^{\prime} \widehat{\otimes} L_{x, y}^{1}
$$


which follows from the characterization of $\mathcal{D}_{L^{1}}^{\prime}$ by regularisation given in Théorème XXV in [20, p. 201]. Using the kernel identities $\mathcal{D}_{x, y}^{\prime}=\mathcal{D}_{x}^{\prime} \widehat{\otimes} \mathcal{D}_{y}^{\prime}$ and $L_{x y}^{1}=L_{x}^{1} \widehat{\otimes}_{\pi} L_{y}^{1}$, we obtain

$$
K(x-z, y-w) \in \mathcal{D}_{z}^{\prime} \widehat{\otimes} \mathcal{D}_{w}^{\prime}\left(L_{x}^{1} \widehat{\otimes}_{\pi} L_{y}^{1}\right)=\left(\mathcal{D}_{z}^{\prime} \widehat{\otimes} L_{x}^{1}\right) \widehat{\otimes}_{\pi}\left(\mathcal{D}_{w}^{\prime} \widehat{\otimes}_{\pi} L_{y}^{1}\right)
$$

Applying Proposition 7 twice to the line above, we finally get

$$
\begin{aligned}
K(x, y) \in \mathcal{D}_{L^{1}, x, y}^{\prime} & \Leftrightarrow K(x-z, y-w) \in\left(\mathcal{D}_{z}^{\prime} \widehat{\otimes} L_{x}^{1}\right) \widehat{\otimes}_{\pi}\left(\mathcal{D}_{w}^{\prime} \widehat{\otimes}_{\pi} L_{y}^{1}\right) \\
& \Leftrightarrow K(x, y) \in \mathcal{D}_{L^{1}, x}^{\prime} \widehat{\otimes}_{\pi} \mathcal{D}_{L^{1}, y}^{\prime},
\end{aligned}
$$

i.e. we have shown the algebraic identity $\mathcal{D}_{L^{1}, x y}^{\prime}=\mathcal{D}_{L^{1}, x}^{\prime} \widehat{\otimes}_{\pi} \mathcal{D}_{L^{1}, y}^{\prime}$.

In order to prove the continuity of the identity mapping

$$
\mathcal{D}_{L^{1}, x}^{\prime} \widehat{\otimes}_{\pi} \mathcal{D}_{L^{1}, y}^{\prime} \rightarrow \mathcal{D}_{L^{1}, x y}^{\prime}
$$

it is sufficient to show the continuity of the bilinear mapping

$$
\mathcal{D}_{L^{1}, x}^{\prime} \times \mathcal{D}_{L^{1}, y}^{\prime} \rightarrow \mathcal{D}_{L^{1}, x y}^{\prime},(S(x), T(y)) \mapsto S(x) \otimes T(y)
$$

The continuity of this mapping follows from the separate continuity due to the fact that for (DF)-spaces separate continuity of bilinear maps implies continuity. The separate continuity follows immediately by the closed graph theorem.

By de Wilde's closed graph theorem (Theorem 5.4.1 in [9, p. 92]) the identity is a topological isomorphism because $\mathcal{D}_{L^{1}, x y}^{\prime}$ is ultrabornological and $\mathcal{D}_{L^{1}, x}^{\prime} \widehat{\otimes}_{\pi} \mathcal{D}_{L^{1}, y}^{\prime}$ is a complete (DF)-space and, hence, has a completing web by Proposition 12.4.6 in [9, p. 260].

Proposition 10 The space of integrable smooth functions satisfies the kernel identity

$$
\mathcal{D}_{L^{1}, x y}=\mathcal{D}_{L^{1}, x} \widehat{\otimes}_{\pi} \mathcal{D}_{L^{1}, y}
$$

algebraically and topologically.

Proof For $S \in \mathcal{D}^{\prime}$ we get

$$
S \in \mathcal{D}_{L^{1}} \Leftrightarrow S(x-y) \in \mathcal{E}_{y} \widehat{\otimes} L_{x}^{1}
$$

and therefore for $K \in \mathcal{D}_{x y}^{\prime}$,

$$
K(x, y) \in \mathcal{D}_{L^{1}, x y} \Leftrightarrow K(x-z, y-w) \in \mathcal{E}_{z w} \widehat{\otimes} L_{x y}^{1} .
$$


From this equivalence, we deduce

$$
\begin{aligned}
K(x, y) \in \mathcal{D}_{L^{1}, x y} & \Leftrightarrow K(x-z, y-w) \in \mathcal{E}_{z} \widehat{\otimes} \mathcal{E}_{w} \widehat{\otimes}\left(L_{x}^{1} \widehat{\otimes}_{\pi} L_{y}^{1}\right) \\
& =\left(\mathcal{E}_{z} \widehat{\otimes} L_{x}^{1}\right) \widehat{\otimes}_{\pi}\left(\mathcal{E}_{w} \widehat{\otimes} L_{y}^{1}\right)
\end{aligned}
$$

using the classical kernel identities $\mathcal{E}_{x y}=\mathcal{E}_{x} \widehat{\otimes} \mathcal{E}_{y}$ and $L_{x y}^{1}=L_{x}^{1} \widehat{\otimes}_{\pi} L_{y}^{1}$. From Proposition 7, applied twice, we get

$$
K(x, y) \in \mathcal{D}_{L^{1}, x y} \Leftrightarrow K(x, y-w) \in \mathcal{D}_{L^{1}, x} \widehat{\otimes}_{\pi}\left(\mathcal{E}_{w} \widehat{\otimes} L_{y}^{1}\right) \Leftrightarrow K(x, y) \in \mathcal{D}_{L^{1}, x y} .
$$

As the $\pi$-tensor product of continuous mappings is continuous, the mapping

$$
\mathcal{D}_{L^{1}, x} \widehat{\otimes}_{\pi} \mathcal{D}_{L^{1}, y} \rightarrow L_{x}^{1} \widehat{\otimes}_{\pi} L_{y}^{1}=L_{x y}^{1}, f \mapsto \partial_{x}^{\alpha} \partial_{y}^{\beta} f
$$

is continuous for all multi-indices $\alpha$ and $\beta$. Hence the $\pi$-topology is finer than the topology of $\mathcal{D}_{L^{1}}$. As these topologies are comparable Fréchet space topologies on the same vector space they coincide by the closed graph theorem.

Acknowledgements Open access funding provided by University of Innsbruck and Medical University of Innsbruck.

Open Access This article is distributed under the terms of the Creative Commons Attribution 4.0 International License (http://creativecommons.org/licenses/by/4.0/), which permits unrestricted use, distribution, and reproduction in any medium, provided you give appropriate credit to the original author(s) and the source, provide a link to the Creative Commons license, and indicate if changes were made.

\section{References}

1. Adams, R.A., Fournier, J.J.F.: Sobolev Spaces, Volume 140 of Pure and Applied Mathematics, 2nd edn. Elsevier/Academic Press, Amsterdam (2003)

2. Bargetz, C.: Completing the Valdivia-Vogt tables of sequence-space representations of spaces of smooth functions and distributions. Monatsh. Math. 177(1), 1-14 (2015)

3. Bargetz, C., Nigsch, E.A., Ortner, N.: Convolvability and regularization of distributions. Annali di Matematica (2017). doi:10.1007/s10231-017-0662-3

4. Bargetz, C., Ortner, N.: Dissertationes mathematicae. In: Convolution of vector-valued distributions: a survey and comparison, vol. 495, p. 51 (2013)

5. Chevalley, C.: Theory of Distributions. Columbia University (1950/51)

6. Grothendieck, A.: Résumé des résultats essentiels dans la théorie des produits tensoriels topologiques et des espaces nucléaires. Ann. Inst. Fourier Grenoble 4, 73-112 (1952)

7. Grothendieck, A.: Produits tensoriels topologiques et espaces nucléaires. Mem. Am. Math. Soc. 1955(16): Chap I, 196, Chap II, 140 (1955)

8. Horváth, J.: Sur la convolution des distributions. Bull. Sci. Math. (2) 98(3), 183-192 (1974)

9. Jarchow, H.: Locally convex spaces. In: Mathematische Leitfäden. B. G. Teubner, Stuttgart (1981)

10. Larcher, J.: Multiplications and convolutions in L. Schwartz' spaces of test functions and distributions and their continuity. Analysis (Berlin) 33(4), 319-332 (2013)

11. Ortner, N.: Sur la convolution des distributions. C. R. Acad. Sci. Paris Sér. A-B 290(12), A533-A536 (1980)

12. Ortner, N.: On convolvability conditions for distributions. Monatsh. Math. 160(3), 313-335 (2010)

13. Ortner, N., Wagner, P.: Distribution-Valued Analytic Functions. Edition SWK, Tredition, Hamburg (2013) 
14. Roider, B.: Sur la convolution des distributions. Bull. Sci. Math. (2) 100(3), 193-199 (1976)

15. Schwartz, L.: Produits tensoriels topologiques d'espaces vectoriels topologiques. Espaces vectoriels topologiques nucléaires. Applications, vol. 1 (1953/1954)

16. Schwartz, L.: Espaces de fonctions différentiables à valeurs vectorielles. J. Anal. Math. 4, 88-148 (1954/1955)

17. Schwartz, L.: Lectures on Mixed Problems in Partial Differential Equations and Representations of Semi-groups. Tata Institute of Fundamental Research, Bombay (1957)

18. Schwartz, L.: Théorie des distributions à valeurs vectorielles. I. Ann. Inst. Fourier Grenoble 7, 1-141 (1957)

19. Schwartz, L.: Théorie des distributions à valeurs vectorielles. II. Ann. Inst. Fourier Grenoble 8, 1-209 (1958)

20. Schwartz, L.: Théorie des distributions. Publications de l'Institut de Mathématique de l'Université de Strasbourg, No. IX-X. Nouvelle édition, entièrement corrigée, refondue et augmentée. Hermann, Paris (1966)

21. Shiraishi, R.: On the definition of convolutions for distributions. J. Sci. Hiroshima Univ. Ser. A 23(1932), 1959 (1959) 